\title{
Free Energy and Dissipation Rate for Reaction Diffusion Processes of Electrically Charged Species
}

\author{
Annegret Glitzky*, Konrad Gröger, Rolf Hünlich \\ Weierstrass Institute for Applied Analysis and Stochastics \\ Mohrenstraße 39 \\ D-10117 Berlin, Germany
}

December 6, 1995

1991 Mathematics Subject Classification. 35B40, 35K45, 35K57,78A35.

Keywords. Reaction-diffusion systems, drift-diffusion processes, motion of charged particles, steady states, asymptotic behaviour.

*Supported by the BMBF under contract 03-GA7FVB-1.0M840. 


\begin{abstract}
The paper deals with a special problem concerning the transport of electrically charged species via diffusion, drift, and reaction mechanisms. We prove for a variety of models that without knowing any a priori estimate for the chemical potentials one can estimate the free energy from above by the corresponding dissipation rate. The inequality presented here can be interpreted as a nonlinear analogue of Korn's Inequality or Poincaré's Inequality. As a consequence of our main result we show that the free energy approximates its equilibrium value exponentially as time tends to infinity.
\end{abstract}

\title{
1. Introduction
}

In this paper we prove that for many models of reaction-diffusion processes of electrically charged species the free energy can be estimated from above by the corresponding dissipation rate. Such an estimate is of interest for several reasons.

First we should mention that reaction-diffusion processes of species some of which are electrically charged take place in many branches of technology, for example in microelectronics.

Next we want to emphazise that by means of our estimate it is easy to show that the free energy approximates its equilibrium value exponentially as time tends to infinity. For this purpose one does not need further upper or lower bounds for concentrations or chemical potentials. On the contrary, the asymptotic behaviour of the free energy can be used as a starting point for a Moser iteration leading to $L^{\infty}$-bounds for the chemical potentials.

Finally we note that the inequality we prove can be seen as a nonlinear analogue of Korn's Inequality and Poincaré's Inequality. To a certain extent the proof of our main result follows the proofs of these inequalities. Let us mention that in the field of mechanics another nonlinear analogue of Korn's Inequality has been proved by Kohn [8].

An estimate of the same kind as the main result of this paper had been proved for reaction-diffusion processes of uncharged species by Gröger [5]. For a special case with only one kind of charged dopants analogous results have been obtained under the so called electroneutrality condition by [2], [3]. Glitzky and Hünlich [4] present a more general result with arbitrarily many charged species. They assume that the relations between chemical potentials and concentrations of the species are governed by Boltzmann statistics. Moreover, they allow reactions to take place in the interior and on the boundary of the domain occupied by the species. In the present paper we generalize these ideas to a broader class of statistics and to more general reaction terms. In addition we include a nonlinear (capacity) term into the Poisson equation for the electrostatic potential. This allows to treat models arising by eliminating some of the species (cf. Höfler, Strecker [7]). Whereas for Boltzmann statistics concentrations and chemical activities are of the same order of magnitude, in the general case treated here this is no longer the case. To overcome the 
difficulties related to this fact we need additional a priori estimates. Because we are able to present satisfactory a priori estimates only for the spatially two-dimensional case we restrict our considerations from the beginning to that case. Our new a priori estimates are essentially based on a well known imbedding result by Trudinger (see [11]).

The paper is organized as follows. In Section 2 we introduce the model equations, we explain the notation adopted in this paper, and we state the basic assumptions with respect to the data of the problem under consideration. In Section 3 we deal with equilibrium states. We introduce a class of sets which are invariant with respect to the transient problem, and we show that each of these classes contains a unique equilibrium state. This section follows closely to the lines of the corresponding section of [4]. In the last section we formulate and prove our main result, the estimate of the free energy by the dissipation rate which leads to the exponential decay of the free energy to its equilibrium value along trajectories of the system.

\section{Model equations, notation, and assumptions}

In this section we describe the mathematical model of the processes we are interested in. Simultaneously, we introduce an appropriate notation and we formulate the assumptions needed in the main part of the paper.

Let $X_{1}, \ldots, X_{m}$ be species, and let $q_{1}, \ldots, q_{m}$ be the specific charges of $X_{1}, \ldots, X_{m}$, respectively, i.e., let

$$
q_{i} \in \mathbb{R}, i=1, \ldots, m,
$$

be the charge of a mass unit of the species $X_{i}, i=1, \ldots, m$.

We assume that $X_{1}, \ldots, X_{m}$ occupy $\bar{\Omega}$, where

$$
\Omega \text { is a bounded Lipschitzian domain in } \mathbb{R}^{2} \text {. }
$$

As mentioned in the introduction, we restrict our considerations to the two-dimensional case because only in this case the results we can prove are satisfactory. As ususal, $H^{1}(\Omega)$ denotes the Sobolev space of square integrable functions on $\Omega$ with square integrable first derivatives. For the norm of a function $v \in H^{1}(\Omega)$ we write $\|v\|_{H^{1}}$. We introduce $D: H^{1}(\Omega) \longrightarrow L^{2}(\Omega)$ by $D u:=\operatorname{grad} u, u \in H^{1}(\Omega)$. Because of this definition the adjoint $D^{*}$ of $D$ maps $L^{2}(\Omega)$ into $\left(H^{1}(\Omega)\right)^{*}$, the dual of $H^{1}(\Omega)$. If $v=\left(v_{0}, \ldots, v_{m}\right)$ then $D v:=\left(D v_{0}, \ldots, D v_{m}\right)$. Unless otherwise specified expressions like "measurable" and "almost every" refer to the standard Lebesgue measure.

The species $X_{1}, \ldots, X_{m}$ take part in chemical reactions. Some of these reactions may be concentrated to the boundary of $\Omega$ or to interfaces between different parts of $\Omega$. In order to treat all reactions in a unified manner we proceed in a way which is not standard but seems to be quite useful. We assume that a measure $\mu$ on $\bar{\Omega}$ is given such that

$\mu$-measurable subsets of $\bar{\Omega}$ are Lebesgue measurable

and

$$
\mu \geq \mu_{\Omega}, \quad \forall u \in H^{1}(\Omega): \exp (u) \in L^{1}(\bar{\Omega}, \mu), \int_{\bar{\Omega}} \exp (u) d \mu \leq \varphi\left(\|u\|_{H^{1}}\right)
$$


for some increasing function $\varphi: \mathbb{R}_{+} \longrightarrow \mathbb{R}_{+}$; here $\mu_{\Omega}$ denotes the Lebesgue measure on $\Omega$. An example of such a measure is $\mu=\mu_{\Omega}+\mu_{\partial \Omega}$, where $\mu_{\partial \Omega}$ is the standard surface measure on $\partial \Omega$ (cf. [11] and Lemma 1.1 in [4]). For results about the imbedding of $H^{1}(\Omega)$ into $L^{p}$ spaces for different measures $\mu$ we refer to Mazja [9]. The norm of $v \in L^{p}(\bar{\Omega}, \mu), 1 \leq p \leq \infty$, will be denoted by $\|v\|_{p, \mu}$. It follows from (A4) that, for $1 \leq p<\infty$,

$$
\forall v \in H^{1}(\Omega):\|v\|_{p, \mu} \leq c_{p}\|v\|_{H^{1}}
$$

As a consequence, each of the spaces $L^{q}(\bar{\Omega}, \mu), 1<q \leq \infty$, can be understood as a subspace of $\left(H^{1}(\Omega)\right)^{*}$. The assumption (A3) and the property $\mu \geq \mu_{\Omega}$ guarantee that each element of $L^{p}(\bar{\Omega}, \mu)$ can be considered as an element of $L^{p}(\Omega)$. Later on we shall exploit this fact tacitly.

The relation between the densities $u_{1}, \ldots, u_{m}$ of the species $X_{1}, \ldots, X_{m}$ and the corresponding chemical potentials $v_{1}, \ldots, v_{m}$ is supposed to be of the form

$$
u_{i}=\bar{u}_{i} g_{i}\left(v_{i}-\bar{v}_{i}\right), \quad i=1, \ldots, m
$$

where

$$
\left.\begin{array}{l}
g_{i} \in C^{1}(\mathbb{R}), \bar{u}_{i} \in L_{+}^{\infty}(\bar{\Omega}, \mu) \backslash\{0\}, \bar{v}_{i} \in L^{\infty}(\bar{\Omega}, \mu), i=1, \ldots, m, \\
\lim _{y \rightarrow \infty} \frac{1}{y} g_{i}(y)=+\infty, 0<\delta \min \left\{1, g_{i}(y)\right\} \leq g_{i}^{\prime}(y) \leq \delta^{-1} g_{i}(y), \\
\delta \min \{1, \exp (y)\} \leq g_{i}(y) \leq \delta^{-1} \exp (y), i=1, \ldots, m, y \in \mathbb{R} .
\end{array}\right\}
$$

The functions $\bar{u}_{i}$ and $\bar{v}_{i}$ are known reference densities and reference potentials, respectively. The fact that the reference values may depend on the spatial position expresses the possible heterogeneity of the system under consideration. The functions $g_{i}$ reflect the underlying statistics. In the case of Boltzmann statistics each $g_{i}$ is the exponential function. Our assumptions with respect to $g_{i}$ are such that all cases of practical interest are included, in particular the Fermi-Dirac statistics. In (A5) and in the sequel $\delta$ denotes an appropriate strictly positive constant, and the subscript + indicates the standard positive cone in a space.

With respect to the electrical field we assume that it is given as $-D v_{0}$, where $v_{0}$ is the electrostatic potential. This is a standard assumption in semiconductor theory. It means that changes of the magnetic field are so slowly that they can be neglected. To describe the flows $j_{1}, \ldots, j_{m}$ of the species $X_{1}, \ldots, X_{m}$ we need the quantities $\zeta_{i}:=q_{i} v_{0}+v_{i}, i=1, \ldots, m$, the so called electrochemical potentials of the species. The gradient $D \zeta_{i}$ is to be interpreted as the driving force for $j_{i}$. In the simplest case $j_{i}$ is proportional to $-u_{i} D \zeta_{i}$. We shall assume that

$$
j_{i}=-d_{i}\left(\cdot, v_{i}, D \zeta_{i}\right)
$$

where $d_{i}$ is a given function with the following properties:

$$
\begin{aligned}
& d_{i}: \Omega \times \mathbb{R} \times \mathbb{R}^{2} \longrightarrow \mathbb{R}^{2} \text { is such that } \\
& d_{i}(x, \cdot, \cdot): \mathbb{R} \times \mathbb{R}^{2} \longrightarrow \mathbb{R}^{2} \text { is continuous for almost every } x \in \Omega, \\
& d_{i}(\cdot, y, \xi): \Omega \longrightarrow \mathbb{R}^{2} \text { is measurable for all } y \in \mathbb{R}, \xi \in \mathbb{R}^{2}, \\
& \delta g_{i}(y)|\xi|^{2} \leq d_{i}(x, y, \xi) \cdot \xi,\left|d_{i}(x, y, \xi)\right| \leq \delta^{-1} g_{i}(y)|\xi| \\
& \text { for almost every } x \in \Omega, \text { for all } y \in \mathbb{R}, \xi \in \mathbb{R}^{2}, i=1, \ldots, m .
\end{aligned}
$$


These assumptions are such that several cases of practical interest are included. We don't allow, however, the flow $j_{i}$ to depend on all gradients $D \zeta_{1}, \ldots, D \zeta_{m}$.

To describe chemical reactions we assume that

$$
\mathcal{R} \subset \mathbb{Z}_{+}^{m} \times \mathbb{Z}_{+}^{m} \text { is a finite subset. }
$$

A pair $(\alpha, \beta) \in \mathcal{R}$ represents the vectors of stoichiometric coefficients of a pair of reactions, usually written in the following form:

$$
\alpha_{1} X_{1}+\cdots+\alpha_{m} X_{m} \rightleftharpoons \beta_{1} X_{1}+\cdots+\beta_{m} X_{m}
$$

We shall assume that the net rate of this pair of reactions is of the form $k_{\alpha \beta}\left(a^{\alpha}-a^{\beta}\right)$, where $k_{\alpha \beta}$ is a reaction coefficient, $a_{i}:=\exp \left(\zeta_{i}\right)$ is the electrochemical activity of $X_{i}$, and $a^{\alpha}:=\prod_{i=1}^{m} a_{i}^{\alpha_{i}}$. The difference of this model to standard mass action kinetics is that concentrations are replaced by activities. This is necessary for the model to be in accordance with the Second Law of Thermodynamics (cf. Othmer [10]). With respect to the reaction coefficients $k_{\alpha \beta},(\alpha, \beta) \in \mathcal{R}$, we require that

$$
k_{\alpha \beta} \in L_{+}^{\infty}(\bar{\Omega}, \mu) \backslash\{0\} \quad \text { for }(\alpha, \beta) \in \mathcal{R} .
$$

Reactions taking place on the boundary $\partial \Omega$ can be described by functions $k_{\alpha \beta}$ supported on $\partial \Omega$. (It was this possibility to treat reactions on the boundary $\partial \Omega$ in the same way as reactions in $\Omega$ which lead us to the introduction of the measure $\mu$.) The net production rate of species $X_{i}$ corresponding to the reaction rates for all reactions taking place is $R_{i}:=\sum_{(\alpha, \beta) \in \mathcal{R}} k_{\alpha \beta}\left(a^{\alpha}-a^{\beta}\right)\left(\beta_{i}-\alpha_{i}\right)$.

The continuity equation for the concentrations taking into account reaction, diffusion, and drift processes can be written as follows:

$$
\frac{\partial u_{i}}{\partial t}-D^{*} j_{i}=R_{i}, i=1, \ldots, m
$$

These equations are to be considered as equations for functions of time with values in $\left(H^{1}(\Omega)\right)^{*}$. Note that $(2.3)$ includes what is usually written as a differential equation in $\Omega$ and a boundary condition on $\partial \Omega$. With our way of writing the continuity equation we want to convince the reader that neither from the physical nor from the mathematical point of view it is necessary to treat separately processes in the interior and on the boundary (or on interfaces) of $\Omega$. By the choice of $\bar{u}_{i}$ we can model a capability of the boundary (or interfaces) to store the species temporarily (cf. Remark 4.7 at the end of this paper).

The Poisson equation satisfied by the electrostatic potential can be written as

$$
D^{*}\left(\varepsilon D v_{0}\right)+e_{0}\left(\cdot, v_{0}\right)=u_{0}:=\sum_{i=1}^{m} q_{i} u_{i}
$$

where $\varepsilon$ is the dielectric permittivity and $e_{0}$ is a function modeling capacities (in the interior or on the boundary of $\Omega$ ). We assume that

$$
\varepsilon \in L^{\infty}(\Omega), \varepsilon \geq \delta,
$$


$e_{0}: \bar{\Omega} \times \mathbb{R} \longrightarrow \mathbb{R}$ is such that

$e_{0}(x, \cdot): \mathbb{R} \longrightarrow \mathbb{R}$ is continuous for $\mu$-almost every $x \in \bar{\Omega}$,

$e_{0}(\cdot, y): \bar{\Omega} \longrightarrow \mathbb{R}$ is $\mu$-measurable for every $y \in \mathbb{R}$,

$\left|e_{0}(x, y)\right| \leq \exp \left(\delta^{-1}(|y|+1)\right), e_{0}(x, y)-e_{0}(x, \eta) \geq \bar{u}_{0}(x)(y-\eta)$

for $y>\eta, x \in \bar{\Omega}$, and some $\bar{u}_{0} \in L_{+}^{\infty}(\bar{\Omega}, \mu) \backslash\{0\}$.

In order to give a more precise formulation of the equations (2.3), (2.4) we introduce the following spaces:

$$
\begin{gathered}
V:=H^{1}\left(\Omega ; \mathbb{R}^{m+1}\right), W:=\left\{v \in V: \exp \left(v_{i}\right) \in L^{\infty}(\Omega), i=1, \ldots, m\right\}, \\
\mathcal{S}:=\operatorname{span}\{\alpha-\beta:(\alpha, \beta) \in \mathcal{R}\}, \quad \mathcal{S}^{\perp}:=\text { orthogonal complement of } \mathcal{S} \text { in } \mathbb{R}^{m} .
\end{gathered}
$$

In addition to $(\mathrm{A} 1)-(\mathrm{A} 10)$ we assume that we are given $u^{0} \in V^{*}$ such that

$$
\left.\begin{array}{l}
u^{0}=\left(u_{0}^{0}, u_{1}^{0}, \ldots, u_{m}^{0}\right), u_{0}^{0}=\sum_{i=1}^{m} q_{i} u_{i}^{0}, u_{i}^{0} \geq 0, i=1, \ldots, m, \\
\sum_{i=1}^{m} \lambda_{i}\left\langle u_{i}^{0}, 1\right\rangle>0 \text { if } \lambda=\left(\lambda_{1}, \ldots, \lambda_{m}\right) \in \mathcal{S}_{+}^{\perp} \backslash\{0\} .
\end{array}\right\}
$$

As usual, $V^{*}$ denotes the space dual to $V$, and 1 means the constant function on $\Omega$ taking the value 1. (Generally we shall use the same notation for a constant function and its value. This should not lead to misunderstandings.) Note that the last assumption with respect to $u^{0}$ is satisfied if $u_{i}^{0} \geq 0, u_{i}^{0} \neq 0, i=1, \ldots, m$. The element $u^{0}$ plays the rôle of an initial value for the vector function $u:=\left(u_{0}, \ldots, u_{m}\right)$.

Next we define operators $L: V \longrightarrow H^{1}\left(\Omega ; \mathbb{R}^{m}\right), A: W \longrightarrow V^{*}$, and $E: V \longrightarrow V^{*}$ as follows:

$$
\begin{aligned}
L v:= & \left(v_{1}+q_{1} v_{0}, \ldots, v_{m}+q_{m} v_{0}\right) \text { for } v=\left(v_{0}, \ldots, v_{m}\right) \in V, \\
\langle A w, v\rangle:= & \int_{\Omega} \sum_{i=1}^{m} d_{i}\left(\cdot, w_{i}, D L_{i} w\right) \cdot D L_{i} v d x \\
& +\int_{\bar{\Omega}} \sum_{(\alpha, \beta) \in \mathcal{R}} k_{\alpha \beta}\left(a^{\alpha}-a^{\beta}\right)(\alpha-\beta) \cdot L v d \mu \text { for } w \in W, v \in V,
\end{aligned}
$$

where $a:=\left(\exp \left(L_{1} w\right), \ldots, \exp \left(L_{m} w\right)\right)$,

$$
\begin{gathered}
\left\langle E_{0} v_{0}, w_{0}\right\rangle:=\int_{\Omega} \varepsilon D v_{0} \cdot D w_{0} d x+\int_{\bar{\Omega}} e_{0}\left(\cdot, v_{0}\right) w_{0} d \mu \text { for } v_{0}, w_{0} \in H^{1}(\Omega), \\
E v:=\left(E_{0} v_{0}, e_{1}\left(\cdot, v_{1}\right), \ldots, e_{m}\left(\cdot, v_{m}\right)\right) \text { for } v \in V
\end{gathered}
$$

where

$$
e_{i}(x, y):=\bar{u}_{i}(x) g_{i}\left(y-\bar{v}_{i}(x)\right) \text { for } x \in \bar{\Omega}, y \in \mathbb{R}, i=1, \ldots, m .
$$

Using (A9) and (A10) one can easily prove that $E_{0}: H^{1}(\Omega) \longrightarrow H^{1}(\Omega)^{*}$ is strongly monotone, i.e., there exists $\gamma>0$ such that

$$
\left\langle E_{0} v_{0}-E_{0} w_{0}, v_{0}-w_{0}\right\rangle \geq \gamma\left\|v_{0}-w_{0}\right\|_{H^{1}}^{2} \text { for } v_{0}, w_{0} \in H^{1}(\Omega) .
$$


Now we are able to write the transient problem (2.1) - (2.4) more precisely as follows: We are looking for functions $u \in H_{\text {loc }}^{1}\left(\mathbb{R}_{+} ; V^{*}\right), v \in L_{l o c}^{2}\left(\mathbb{R}_{+} ; V\right) \cap L_{l o c}^{\infty}\left(\mathbb{R}_{+} ; W\right)$ such that

$$
\frac{d u}{d t}(t)+A v(t)=0, u(t)=E v(t) \text { for a.e. } t \in \mathbb{R}_{+}, u(0)=u^{0} .
$$

For $v \in V($ and $\zeta:=L v)$ the value

$$
\rho(v):=\int_{\Omega} \sum_{i=1}^{m} d_{i}\left(\cdot, v_{i}, D \zeta_{i}\right) \cdot D \zeta_{i} d x+\int_{\bar{\Omega}} \sum_{(\alpha, \beta) \in \mathcal{R}} k_{\alpha \beta}\left(e^{\zeta \cdot \alpha}-e^{\zeta \cdot \beta}\right)(\alpha-\beta) \cdot \zeta d \mu
$$

(which may be $+\infty$ ) will be called the dissipation rate associated to $v$. The reason for this terminology is the following. If $(u, v)$ is a solution to the initial value problem $(2.13)$ then

$$
\rho(v(t))=\langle A v(t), v(t)\rangle=-\left\langle\frac{d u}{d t}(t), v(t)\right\rangle,
$$

and in thermodynamics this expression is the dissipation rate of the process governed by (2.13) at time $t$.

To define the free energy of a state of the system under consideration we first introduce a functional $G: V \longrightarrow \mathbb{R}$ as follows:

$$
G(v):=\int_{\Omega} \frac{\varepsilon}{2}\left|D v_{0}\right|^{2} d x+\sum_{i=0}^{m} \int_{\bar{\Omega}} \int_{0}^{v_{i}} e_{i}(\cdot, y) d y d \mu .
$$

It is easy to check that $G$ is convex and that $G^{\prime}=E$, i.e., the operator $E$ (cf. (2.9) (2.11)) is the Gâteaux derivative of $G$. The conjugate of the functional $G$ will be denoted by $F$. For $u \in V^{*}$ the value $F(u)$ is to be interpreted as the free energy of the state $u$. It is given by

$$
F(u):=\sup _{v \in V}\{\langle u, v\rangle-G(v)\} .
$$

As mentioned in the introduction we are mainly interested in a relation between the free energy and the dissipation rate. To describe this relation we need some information about stationary solutions to $(2.13)$.

\section{Equilibria}

First we define

$$
U:=\left\{u \in V^{*}: u_{0}=\sum_{i=1}^{m} q_{i} u_{i},\left(\left\langle u_{1}, 1\right\rangle, \ldots,\left\langle u_{m}, 1\right\rangle\right) \in \mathcal{S}\right\} .
$$

The importance of $U$ lies in the fact that $u(t)-u^{0} \in U$ for every $t>0$ if $(u, v)$ is a solution to (2.13). Hence, if $u^{*}:=\lim _{t \rightarrow \infty} u(t)$ exists, then we have necessarily $u^{*} \in U+u^{0}$. 
Remark 3.1. It is easy to check that the set $U^{\perp}:=\{v \in V:\langle u, v\rangle=0$ for every $u \in U\}$ can be characterized as follows:

$$
U^{\perp}=\left\{v \in V: D L v=0, L v \in \mathcal{S}^{\perp}\right\}
$$

This will frequently be used throughout the paper.

Theorem 3.2. There exists a unique $v^{*} \in W$ such that $A v^{*}=0$ and $u^{*}:=E v^{*} \in U+u^{0}$. It holds $D L v^{*}=0$ and $L v^{*} \in \mathcal{S}^{\perp}$.

Before we prove this theorem we present an auxiliary result.

In the next lemma $I_{U^{\perp}}$ denotes the indicator functional of $U^{\perp}$, i.e., the functional vanishing on $U^{\perp}$ and taking the value $+\infty$ on $V \backslash U^{\perp}$.

Lemma 3.3. The functional $G_{0}:=G+I_{U^{\perp}}-u^{0}$ is proper, convex, and lower semicontinuous. It satisfies $\lim _{\|v\|_{V} \rightarrow \infty} G_{0}(v)=+\infty$.

Proof. Only the last assertion needs to be proved, the other properties of $G_{0}$ are evident. It is easy to check that, for $v \in U^{\perp}$,

$$
G_{0}(v) \geq \delta\left\|v_{0}\right\|_{H^{1}}^{2}+\sum_{i=1}^{m}\left\|\bar{u}_{i} v_{i}^{+}\right\|_{1, \mu}-c .
$$

Here and in the sequel $c$ denotes (not necessarily equal) constants the value of which is not important and the superscript + indicates the positive part of a function. By (3.2) it suffices to show that $\lim _{n \rightarrow \infty} G_{0}\left(v_{n}\right)=+\infty$ provided that $v_{n} \in U^{\perp},\left\|v_{n 0}\right\|_{H^{1}} \leq c$, $\left\|\bar{u}_{i} v_{n i}^{+}\right\|_{1, \mu} \leq c, i=1, \ldots, m$, and $\left\|v_{n}\right\|_{V} \longrightarrow \infty$. Let $\zeta_{n}:=L v_{n}$. Then $D \zeta_{n}=0$ and $\zeta_{n} \in \mathcal{S}^{\perp}$ (cf. Remark 3.1). In view of $\left\|v_{n}\right\|_{V} \longrightarrow \infty$ and $\left\|v_{n 0}\right\|_{H^{1}} \leq c$ it holds $\left|\zeta_{n}\right| \longrightarrow \infty$. On the other hand $\zeta_{n i}^{+} \leq v_{n i}^{+}+\left(q_{i} v_{n 0}\right)^{+}$implies that $\zeta_{n i}^{+} \leq c$. Without loss of generality we may assume that $-\frac{\zeta_{n}}{\left|\zeta_{n}\right|} \longrightarrow \lambda$ in $\mathbb{R}^{m}$. Then $\lambda \in \mathcal{S}_{+}^{\perp} \backslash\{0\}$, and by means of (A11) and the boundedness properties of $\left(v_{n}\right)$ we derive from the definition of $G_{0}$ that

$$
\liminf _{n \rightarrow \infty} \frac{1}{\left|\zeta_{n}\right|} G_{0}\left(v_{n}\right) \geq \sum_{i=1}^{m} \lambda_{i}\left\langle u_{i}^{0}, 1\right\rangle>0 .
$$

This is possible only if $\lim _{n \rightarrow \infty} G_{0}\left(v_{n}\right)=+\infty$.

Proof of Theorem 3.2.

1. Let $v^{*}$ be such that $G_{0}\left(v^{*}\right)$ is the minimal value of $G_{0}$. (By Lemma 3.3 such $v^{*}$ exists.) Then $0 \in \partial G_{0}\left(v^{*}\right)$, where $\partial G_{0}$ denotes the subdifferential of $G_{0}$. We have

$$
\partial G_{0}=E+\partial I_{U^{\perp}}-u^{0}, \partial I_{U^{\perp}}(v)=U \text { for } v \in U^{\perp} .
$$


Since necessarily $v^{*} \in U^{\perp}$ we find $0=E v^{*}+u-u^{0}$ for some $u \in U$. Consequently,

$$
\begin{aligned}
E_{0} v_{0}^{*} & =u_{0}^{0}-u_{0}=\sum_{i=1}^{m} q_{i}\left(u_{i}^{0}-u_{i}\right) \\
& =\sum_{i=1}^{m} q_{i} e_{i}\left(\cdot, v_{i}^{*}\right)=\sum_{i=1}^{m} q_{i} e_{i}\left(\cdot, \zeta_{i}^{*}-q_{i} v_{0}^{*}\right),
\end{aligned}
$$

where $\zeta^{*}=\left(\zeta_{1}^{*}, \ldots, \zeta_{m}^{*}\right):=L v^{*}$. Standard arguments (test functions like $\left.\left(v_{0}^{*}-k\right)^{+}\right)$show that $v_{0}^{*} \in L^{\infty}(\bar{\Omega}, \mu)$. This implies that $v_{i}^{*}=\zeta_{i}^{*}-q_{i} v_{0}^{*} \in L^{\infty}(\bar{\Omega}, \mu), i=1, \ldots, m$.

2. Because of $D \zeta^{*}=0$ and $\zeta^{*} \in \mathcal{S}^{\perp}$ we obtain, for every $v \in V$,

$$
\left\langle A v^{*}, v\right\rangle=\int_{\Omega} \sum_{i=1}^{m} d_{i}\left(\cdot, v_{i}^{*}, 0\right) \cdot D L_{i} v d x+\sum_{(\alpha, \beta) \in \mathcal{R}} \int_{\bar{\Omega}} k_{\alpha \beta}\left(e^{\zeta^{*} \cdot \alpha}-e^{\zeta^{*} \cdot \beta}\right)(\alpha-\beta) \cdot L v d \mu=0 .
$$

Consequently, $A v^{*}=0$.

3. Let $A v=0$ and $E v \in U+u^{0}$ for some $v \in V$, and let $\zeta:=L v$. Then

$$
0=\langle A v, v\rangle=\int_{\Omega} \sum_{i=1}^{m} d_{i}\left(\cdot, v_{i}, D \zeta_{i}\right) \cdot D \zeta_{i} d x+\sum_{(\alpha, \beta) \in \mathcal{R}} \int_{\bar{\Omega}} k_{\alpha \beta}\left(e^{\zeta \cdot \alpha}-e^{\zeta \cdot \beta}\right)(\alpha-\beta) \cdot \zeta d \mu .
$$

In view of (A6) and (A8) we obtain $D \zeta=0$ and, for $(\alpha, \beta) \in \mathcal{R}$,

$$
k_{\alpha \beta}\left(e^{\zeta \cdot \alpha}-e^{\zeta \cdot \beta}\right)(\alpha-\beta) \cdot \zeta=0 .
$$

This is possible only if $\zeta \in \mathcal{S}^{\perp}$. With $v^{*}$ as before we have

$$
\left\langle E v-E v^{*}, v-v^{*}\right\rangle=0
$$

because $E v-E v^{*} \in U$ and $v-v^{*} \in U^{\perp}$. Hence (cf. the definition of $E$ )

$$
\begin{aligned}
0= & \int_{\Omega} \varepsilon\left|D\left(v_{0}-v_{0}^{*}\right)\right|^{2} d x+\int_{\bar{\Omega}}\left(e_{0}\left(\cdot, v_{0}\right)-e_{0}\left(\cdot, v_{0}^{*}\right)\right)\left(v_{0}-v_{0}^{*}\right) d \mu \\
& +\sum_{i=1}^{m} \int_{\bar{\Omega}} \bar{u}_{i}\left(g_{i}\left(v_{i}-\bar{v}_{i}\right)-g_{i}\left(v_{i}^{*}-\bar{v}_{i}\right)\right)\left(v_{i}-v_{i}^{*}\right) d \mu .
\end{aligned}
$$

Because of the properties of $e_{0}$ and $g_{1}, \ldots, g_{m}$ required in (A10) and (A5), respectively, this leads first to $v_{0}=v_{0}^{*}$ and then to $v_{i}(x)=v_{i}^{*}(x), i=1, \ldots, m$, for $x \in \Omega_{i}$, where $\Omega_{i} \subset \Omega$ is such that $\mu\left(\Omega_{i}\right)>0$. Taking into account that, for $i=1, \ldots, m$, the functions $\zeta_{i}:=v_{i}+q_{i} v_{0}$ and $\zeta_{i}^{*}:=v_{i}^{*}+q_{i} v_{0}^{*}$ are constant we find that $v_{i}=v_{i}^{*}, i=1, \ldots, m$. Consequently, the desired result $v=v^{*}$ is true.

Lemma 3.4. If $v^{*}$ is the minimal point of $G_{0}$ then $u^{*}:=E v^{*}$ is the unique minimal point of $\left.F\right|_{U+u^{0}}$

Proof. 1. If $u \in U+u^{0}$ then

$$
\begin{aligned}
F(u)-F\left(u^{*}\right) & =F(u)-\left\langle u^{*}, v^{*}\right\rangle+G\left(v^{*}\right) \\
& =F(u)-\left\langle u, v^{*}\right\rangle+G\left(v^{*}\right) \geq 0 .
\end{aligned}
$$


Here we have used the definition of $F$ and the fact that $u-u^{*} \in U$ and $v^{*} \in U^{\perp}$.

2. If $u \in U+u^{0}$ and $F(u)=F\left(u^{*}\right)$ then

$$
\left\langle u, v^{*}\right\rangle=\left\langle u^{*}, v^{*}\right\rangle=F\left(u^{*}\right)+G\left(v^{*}\right)=F(u)+G\left(v^{*}\right) .
$$

This equality is known to be equivalent to $u \in \partial G\left(v^{*}\right)$. Hence, $u=E v^{*}=u^{*}$.

Lemma 3.5. Let $u=E v \in U+u^{0}$. Then, for some $\gamma>0$,

$$
F(u)-F\left(u^{*}\right) \geq \gamma\left\|v_{0}-v_{0}^{*}\right\|_{H^{1}}^{2}+\gamma \sum_{i=1}^{m}\left\|\sqrt{u_{i}}-\sqrt{u_{i}^{*}}\right\|_{2, \mu}^{2} .
$$

Proof. Under the hypotheses of the lemma we have

$$
\begin{aligned}
F(u)-F\left(u^{*}\right) & =\langle u, v\rangle-G(v)-\left\langle u^{*}, v^{*}\right\rangle+G\left(v^{*}\right) \\
& =\left\langle u, v-v^{*}\right\rangle-G(v)+G\left(v^{*}\right) \\
& =\int_{\Omega} \frac{\varepsilon}{2}\left|D\left(v_{0}-v_{0}^{*}\right)\right|^{2} d x+\int_{\bar{\Omega}} \sum_{i=0}^{m} \int_{v_{i}^{*}}^{v_{i}}\left(e_{i}\left(\cdot, v_{i}\right)-e_{i}(\cdot, y)\right) d y d \mu \\
& \geq \gamma\left\|v_{0}-v_{0}^{*}\right\|_{H^{1}}^{2}+\int_{\bar{\Omega}} \sum_{i=1}^{m} \bar{u}_{i} \int_{v_{i}^{*}}^{v_{i}}\left(g_{i}\left(v_{i}-\bar{v}_{i}\right)-g_{i}\left(y-\bar{v}_{i}\right)\right) d y d \mu .
\end{aligned}
$$

Moreover,

$$
\begin{aligned}
\int_{v_{i}^{*}}^{v_{i}}\left(g_{i}\left(v_{i}-\bar{v}_{i}\right)-g_{i}\left(y-\bar{v}_{i}\right)\right) d y & \geq \delta \int_{v_{i}^{*}}^{v_{i}}\left(\frac{g_{i}\left(v_{i}-\bar{v}_{i}\right)}{g_{i}\left(y-\bar{v}_{i}\right)}-1\right) g_{i}^{\prime}\left(y-\bar{v}_{i}\right) d y \\
& =\delta\left\{g_{i}\left(v_{i}-\bar{v}_{i}\right) \log \frac{g_{i}\left(v_{i}-\bar{v}_{i}\right)}{g_{i}\left(v_{i}^{*}-\bar{v}_{i}\right)}-g_{i}\left(v_{i}-\bar{v}_{i}\right)+g_{i}\left(v_{i}^{*}-\bar{v}_{i}\right)\right\} \\
& \geq \delta\left|\sqrt{g_{i}\left(v_{i}-\bar{v}_{i}\right)}-\sqrt{g_{i}\left(v_{i}^{*}-\bar{v}_{i}\right)}\right|^{2} .
\end{aligned}
$$

Hence, the assertion (3.7) holds. In the preceding estimate we used the elementary relation

$$
\xi \log \frac{\xi}{\eta}-\xi+\eta \geq(\sqrt{\xi}-\sqrt{\eta})^{2} \text { for } \xi, \eta>0 .
$$

Remark 3.6. The proof of Lemma 3.5 shows that, for $u=E v \in U+u^{0}$,

$$
\int_{\bar{\Omega}} \sum_{i=1}^{m} u_{i} \log \left(u_{i}\right) d \mu \leq c(F(u)+1) .
$$

Lemma 3.7. Let $u=E v \in U+u^{0}$. Then

$$
F(u)-F\left(u^{*}\right) \leq c\left\|v_{0}-v_{0}^{*}\right\|_{H^{1}}^{2}+c \sum_{i=1}^{m}\left\|u_{i}-u_{i}^{*}\right\|_{2, \mu}^{2}+\int_{\bar{\Omega}}\left(v_{0}-v_{0}^{*}\right)\left(e_{0}\left(\cdot, v_{0}\right)-e_{0}\left(\cdot, v_{0}^{*}\right)\right) d \mu .
$$


This lemma can be proved similarly as the preceding one. Therefore, we omit the details.

\section{Estimation of the Free Energy}

Let

$$
\left.\begin{array}{rl}
\mathcal{M}:= & \left\{u \in U+u^{0}: \text { It exists } a \in \partial \mathbb{R}_{+}^{m} \text { such that } a^{\alpha}=a^{\beta} \text { for }(\alpha, \beta) \in \mathcal{R}\right. \\
& \text { and } \left.u_{i}=e_{i}\left(\cdot, \log a_{i}-q_{i} E_{0}^{-1} u_{0}\right) \text { if } a_{i}>0, u_{i}=0 \text { else, } i=1, \ldots, m\right\}
\end{array}\right\}
$$

and

$$
R_{\mathcal{M}}:=\inf _{u \in \mathcal{M}} F(u) \quad\left(R_{\mathcal{M}}=+\infty \text { if } \mathcal{M}=\emptyset\right)
$$

Remark 4.1. Obviously, $\mathcal{M}=\emptyset$ if there is no $a \in \partial \mathbb{R}_{+}^{m}$ such that $a^{\alpha}=a^{\beta}$ for all $(\alpha, \beta) \in \mathcal{R}$. But even if there exists such $a \in \partial \mathbb{R}_{+}^{m}$ it may happen that there is no $u$ in $U+u^{0}$ such that $u_{i}=0 \Longleftrightarrow a_{i}=0$. In that case the set $\mathcal{M}$ is empty as well.

Theorem 4.2. Let (A1) - (A11) be satisfied. Moreover, let $R<R_{\mathcal{M}}$ be fixed, and let $u^{*}$ be the same as in the preceding section. Then there exists a constant $C$ such that

$$
F(u)-F\left(u^{*}\right) \leq C \rho(v)
$$

provided that $v \in V, u=E v \in U+u^{0}$, and $F(u) \leq R$.

Proof. 1. If $v \in V, \zeta=L v$, and $a=\left(\exp \left(\zeta_{1}\right), \ldots, \exp \left(\zeta_{m}\right)\right)$, then

$$
\begin{aligned}
\rho(v) & =\int_{\Omega} \sum_{i=1}^{m} d_{i}\left(\cdot, v_{i}, D \zeta_{i}\right) \cdot D \zeta_{i} d x+\int_{\bar{\Omega}} \sum_{(\alpha, \beta) \in \mathcal{R}} k_{\alpha \beta}\left(a^{\alpha}-a^{\beta}\right)(\alpha-\beta) \cdot \zeta d \mu \\
& \geq \int_{\Omega} \delta \sum_{i=1}^{m} g_{i}\left(v_{i}\right)\left|D \zeta_{i}\right|^{2} d x+\int_{\bar{\Omega}} \sum_{(\alpha, \beta) \in \mathcal{R}} k_{\alpha \beta}\left(a^{\alpha / 2}-a^{\beta / 2}\right)^{2} d \mu=: \rho_{1}(v) .
\end{aligned}
$$

Therefore it is sufficient to prove that, under the hypotheses of the theorem,

$$
F(u)-F\left(u^{*}\right) \leq C \rho_{1}(v)
$$

2. We assume (4.3) to be false. Then we can find $u_{n} \in U+u^{0}, v_{n} \in V, n \in \mathbb{N}$, such that

$$
u_{n}=E v_{n}, F\left(u_{n}\right) \leq R, F\left(u_{n}\right)-F\left(u^{*}\right)=C_{n} \rho_{1}\left(v_{n}\right)>0
$$

where $\lim _{n \rightarrow \infty} C_{n}=+\infty$. Lemma 3.5, Remark 3.6 and the results of [6] show that

$$
\left\|v_{n 0}\right\|_{H^{1}}+\left\|v_{n 0}\right\|_{\infty, \mu}+\sum_{i=1}^{m}\left\|u_{n i}\right\|_{1, \mu} \leq c .
$$


3. Let $\zeta_{n}:=L v_{n}$. Then

$$
\begin{aligned}
\left\|D \zeta_{n i}^{+}\right\|_{2}^{2} & \leq \int_{\Omega} \frac{g_{i}\left(\zeta_{n i}^{+}\right)}{g_{i}(0)}\left|D \zeta_{n i}\right|^{2} d x \\
& \leq c \int_{\Omega} g_{i}\left(v_{n i}\right)\left|D \zeta_{n i}\right|^{2} d x \leq c \rho_{1}\left(v_{n}\right) \\
& \leq \frac{c}{C_{n}}\left(R-F\left(u^{*}\right)\right) \longrightarrow 0 \text { as } n \longrightarrow \infty
\end{aligned}
$$

At points $x$, where $\bar{u}_{i}(x) \neq 0$ we have

$$
\left|\bar{u}_{i} \zeta_{n i}^{+}\right| \leq\left|\bar{u}_{i} \zeta_{n i}\right| \leq c \bar{u}_{i}\left|g_{i}^{-1}\left(\frac{u_{n i}}{\bar{u}_{i}}\right)+\bar{v}_{i}+q_{i} v_{n 0}\right| \leq c\left(1+u_{n i}\right)
$$

Hence $\left\|\bar{u}_{i} \zeta_{n i}^{+}\right\|_{1, \mu} \leq c$ and $\left\|\zeta_{n i}^{+}\right\|_{H^{1}} \leq c\left(\right.$ cf. (4.5) and (4.6)). Setting $a_{n i}:=\exp \left(\zeta_{n i}\right)$ we obtain by Trudinger's imbedding theorem that

$$
\left\|a_{n i}\right\|_{p} \leq\left\|1+\exp \left(\zeta_{n i}^{+}\right)\right\|_{p} \leq c_{p}, i=1, \ldots, m, p \in[1, \infty[.
$$

Using (A5) and (4.5) we find that

$$
\frac{a_{n i}}{\sqrt{g_{i}\left(v_{n i}\right)}} \leq c \frac{\exp \left(\zeta_{n i}\right)}{\sqrt{g_{i}\left(\zeta_{n i}\right)}} \leq c\left(a_{n i}+1\right)
$$

Hence

$$
\left\|D a_{n i}\right\|_{r}=\left\|a_{n i} D \zeta_{n i}\right\|_{r} \leq c\left\|a_{n i}+1\right\|_{p}\left\|\sqrt{g_{i}\left(v_{n i}\right)} D \zeta_{n i}\right\|_{2} \leq c \rho_{1}\left(v_{n}\right),
$$

provided that $\frac{1}{r}=\frac{1}{2}+\frac{1}{p}$. The right hand side of (4.7) converges to 0 as $n \longrightarrow \infty$ (cf. (4.6)). Passing to a subsequence if necessary we may assume that

$$
a_{n} \longrightarrow a \text { in } W^{1, r}\left(\Omega ; \mathbb{R}^{m}\right), r \in\left[1,2\left[, \quad v_{n 0} \longrightarrow v_{0} \text { in } H^{1}(\Omega),\right.\right.
$$

where $D a=0$. In addition we may assume that the sequence $\left(a_{n}\right)$ converges pointwise almost everywhere (with respect to $\mu$ ) to $a$. It is easy to check that

$$
\left(a_{n}^{\alpha / 2}-a_{n}^{\beta / 2}\right)^{2} \longrightarrow\left(a^{\alpha / 2}-a^{\beta / 2}\right)^{2}
$$

in $W^{1, r}(\Omega)$, if $r<2$. Therefore,

$$
\int_{\bar{\Omega}} k_{\alpha \beta}\left(a_{n}^{\alpha / 2}-a_{n}^{\beta / 2}\right)^{2} d \mu \longrightarrow \int_{\bar{\Omega}} k_{\alpha \beta} d \mu\left(a^{\alpha / 2}-a^{\beta / 2}\right)^{2} .
$$

Since, for $(\alpha, \beta) \in \mathcal{R}$,

$$
0 \leq \int_{\bar{\Omega}} k_{\alpha \beta}\left(a_{n}^{\alpha / 2}-a_{n}^{\beta / 2}\right)^{2} d \mu \leq \rho_{1}\left(v_{n}\right) \leq \frac{1}{C_{n}}\left(R-F\left(u^{*}\right)\right) \longrightarrow 0
$$

we have necessarily

$$
\forall(\alpha, \beta) \in \mathcal{R}: \quad a^{\alpha}=a^{\beta} .
$$


4. We define

$$
u_{i}:=e_{i}\left(\cdot, \log \left(a_{i}\right)-q_{i} v_{0}\right) \text { if } a_{i} \neq 0, \quad u_{i}:=0 \text { if } a_{i}=0
$$

If $u_{i} \neq 0$ then

$$
\begin{aligned}
\left|u_{n i}-u_{i}\right| & \leq c\left|g_{i}\left(\log \left(a_{n i}\right)-q_{i} v_{n 0}-\bar{v}_{i}\right)-g_{i}\left(\log \left(a_{i}\right)-q_{i} v_{0}-\bar{v}_{i}\right)\right| \\
& \leq c\left|\exp \left(\log \left(a_{n i}\right)-q_{i} v_{n 0}-\bar{v}_{i}\right)-\exp \left(\log \left(a_{i}\right)-q_{i} v_{0}-\bar{v}_{i}\right)\right| \\
& \leq c\left(\left|a_{n i}-a_{i}\right|+\left(a_{n i}+1\right)\left|v_{n 0}-v_{0}\right|\right) .
\end{aligned}
$$

This kind of estimate for $\left|u_{n i}-u_{i}\right|$ is true also if $u_{i}=0$. Since the right hand side of (4.10) converges to 0 in $L^{p}(\bar{\Omega}, \mu)$ for every finite $p$ as $n$ tends to $\infty$, we have

$$
u_{n i} \longrightarrow u_{i} \text { in } L^{p}(\bar{\Omega}, \mu), 1 \leq p<\infty .
$$

5. Next we define $u_{0}:=\sum_{i=1}^{m} q_{i} u_{i}$ and $u:=\left(u_{0}, u_{1}, \ldots, u_{m}\right)$. Starting from $u_{n} \in U+u^{0}$ and $E_{0} v_{n 0}=u_{n 0}$ we obtain in passing to the limit

$$
u \in U+u^{0}, \quad E_{0} v_{0}=u_{0} .
$$

The operator $E_{0}^{-1}: H^{1}(\Omega)^{*} \longrightarrow H^{1}(\Omega)$ being the inverse of a strongly monotone operator is Lipschitzian. Therefore, the sequence $\left(v_{n 0}\right)=\left(E_{0}^{-1} u_{n 0}\right)$ converges strongly in $H^{1}(\Omega)$ to $v_{0}$. Moreover, due to the lower semicontinuity of $F$ on $V^{*}$,

$$
F(u) \leq \liminf _{n \rightarrow \infty} F\left(u_{n}\right) \leq R<R_{\mathcal{M}}
$$

Therefore, $u \notin \mathcal{M}$ (cf. (4.1),(4.2)). This is possible only if $a_{i}>0, i=1, \ldots, m$. Setting $\zeta_{i}:=\log \left(a_{i}\right), v_{i}:=\zeta_{i}-q_{i} v_{0}, i=1, \ldots, m$, we get $v:=\left(v_{0}, v_{1}, \ldots, v_{m}\right) \in V, u=E v \in U+u^{0}$, and $A v=0$. By Theorem 3.2 we conclude that $v=v^{*}$ and $u=u^{*}$.

6. In view of the convergence properties of the sequences $\left(v_{n 0}\right)$ and $\left(u_{n}\right)$ we have (cf. Lemma 3.5, Lemma 3.7)

$$
\lambda_{n}:=\sqrt{F\left(u_{n}\right)-F\left(u^{*}\right)} \longrightarrow 0 \text { as } n \longrightarrow \infty .
$$

Furthermore (cf. (4.4)),

$$
\frac{1}{C_{n}}=\frac{1}{\lambda_{n}^{2}} \rho_{1}\left(v_{n}\right) \geq \int_{\Omega} \delta g_{i}\left(v_{n i}\right)\left|\frac{D \zeta_{n i}}{\lambda_{n}}\right|^{2} d x+\int_{\bar{\Omega}} \sum_{(\alpha, \beta) \in \mathcal{R}} \frac{k_{\alpha \beta}}{\lambda_{n}^{2}}\left(a_{n}^{\alpha / 2}-a_{n}^{\beta / 2}\right)^{2} d \mu .
$$

We introduce

$$
\tilde{v}_{n 0}:=\frac{1}{\lambda_{n}}\left(v_{n 0}-v_{0}\right), \tilde{u}_{n}:=\frac{1}{\lambda_{n}}\left(u_{n}-u\right), b_{n i}:=\frac{1}{\lambda_{n}}\left(\sqrt{\frac{a_{n i}}{a_{i}}}-1\right), i=1, \ldots, m .
$$

Lemma 3.5 shows that $\left(\tilde{v}_{n 0}\right)$ is bounded in $H^{1}(\Omega)$ and that

$$
\begin{aligned}
\left\|\tilde{u}_{n i}\right\|_{3 / 2, \mu} & =\left\|\frac{1}{\lambda_{n}}\left(\sqrt{u_{n i}}-\sqrt{u_{i}}\right)\left(\sqrt{u_{n i}}+\sqrt{u_{i}}\right)\right\|_{3 / 2, \mu} \\
& \leq\left\|\frac{1}{\lambda_{n}}\left(\sqrt{u_{n i}}-\sqrt{u_{i}}\right)\right\|_{2, \mu}\left\|\left(\sqrt{u_{n i}}+\sqrt{u_{i}}\right)\right\|_{6, \mu} \leq c .
\end{aligned}
$$


At points $x$ where $\bar{u}_{i}(x) \neq 0$ we have

$$
\begin{aligned}
\left|a_{n i}-a_{i}\right| & =\left|\exp \left(g_{i}^{-1}\left(\frac{u_{n i}}{\bar{u}_{i}}\right)+\bar{v}_{i}+q_{i} v_{n 0}\right)-\exp \left(g_{i}^{-1}\left(\frac{u_{i}}{\bar{u}_{i}}\right)+\bar{v}_{i}+q_{i} v_{0}\right)\right| \\
& \leq c\left(a_{n i}+1\right) \frac{\left|u_{n i}-u_{i}\right|}{\bar{u}_{i}}+c\left|v_{n 0}-v_{0}\right| .
\end{aligned}
$$

Therefore,

$$
\left\|\bar{u}_{i} b_{n i}\right\|_{1, \mu} \leq c\left\|a_{n i}+1\right\|_{3, \mu}\left\|\tilde{u}_{n i}\right\|_{3 / 2, \mu}+c\left\|\tilde{v}_{n 0}\right\|_{H^{1}}
$$

Using

$$
D b_{n i}=\frac{1}{2 \lambda_{n}} \sqrt{\frac{a_{n i}}{a_{i}}} D \zeta_{n i}=\frac{1}{2 \lambda_{n}} \sqrt{\frac{a_{n i}}{a_{i} g_{i}\left(v_{n i}\right)}} \sqrt{g_{i}\left(v_{n i}\right)} D \zeta_{n i}
$$

and

$$
\frac{a_{n i}}{g_{i}\left(v_{n i}\right)} \leq c \frac{a_{n i}}{g_{i}\left(\zeta_{n i}\right)} \leq c\left(a_{n i}+1\right)
$$

we find

$$
\left\|D b_{n i}\right\|_{r} \leq c\left\|a_{n i}+1\right\|_{p}\left\|\frac{\sqrt{g_{i}\left(v_{n i}\right)} D \zeta_{n i}}{\lambda_{n}}\right\|_{2} \leq \frac{c}{C_{n}}
$$

provided that $\frac{1}{r}=\frac{1}{2}+\frac{1}{p}$. By means of (4.10) we obtain

$$
\left|\tilde{u}_{n i}\right| \leq c\left|\frac{a_{n i}-a_{i}}{\lambda_{n}}\right|+c\left(a_{n i}+1\right)\left|\tilde{v}_{n 0}\right| \leq c\left(\sqrt{a_{n i}}+\sqrt{a_{i}}\right)\left|b_{n i}\right|+c\left(a_{n i}+1\right)\left|\tilde{v}_{n 0}\right| .
$$

The preceding estimates show that, passing to a subsequence if necessary, we may assume that

$$
\begin{gathered}
b_{n i} \longrightarrow b_{i} \text { in } W^{1, r}(\Omega), r<2, \\
\tilde{v}_{n 0} \longrightarrow \tilde{v}_{0} \text { in } H^{1}(\Omega), \tilde{u}_{n} \longrightarrow \tilde{u} \text { in } L^{p}\left(\bar{\Omega}, \mu ; \mathbb{R}^{m+1}\right), 1 \leq p<\infty,
\end{gathered}
$$

and that the sequences $\left(b_{n i}\right),\left(\tilde{v}_{n 0}\right)$ converge pointwise almost everywhere with respect to $\mu$ in $\bar{\Omega}$.

7. In view of $u_{n} \in U+u^{0}$ we have $\frac{1}{\lambda_{n}}\left(u_{n}-u\right) \in U$. Passing to the limit we find that $\tilde{u} \in U$. In particular,

$$
\left(\int_{\bar{\Omega}} \tilde{u}_{1} d \mu, \ldots, \int_{\bar{\Omega}} \tilde{u}_{m} d \mu\right) \in \mathcal{S} \text {. }
$$

On account of the definition of $b_{n i}$ we have, for $(\alpha, \beta) \in \mathcal{R}$,

$$
\left.\begin{array}{l}
a^{-\alpha}\left(a_{n}^{\alpha / 2}-a_{n}^{\beta / 2}\right)^{2}=\left(\prod_{i=1}^{m}\left(\lambda_{n} b_{n i}+1\right)^{\alpha_{i}}-\prod_{i=1}^{m}\left(\lambda_{n} b_{n i}+1\right)^{\beta_{i}}\right)^{2} \\
=\left(\lambda_{n} \sum_{i=1}^{m} b_{n i}\left(\alpha_{i}-\beta_{i}\right)\right)^{2}+Q_{n},
\end{array}\right\}
$$

where

$$
\left|Q_{n}\right| \leq c \lambda_{n}^{3}\left(\left|b_{n}\right|+1\right)^{p_{0}}, \quad 0 \leq p_{0} \leq 2 \max _{(\alpha, \beta) \in \mathcal{R}} \max \left\{\sum_{i=1}^{m} \alpha_{i}, \sum_{i=1}^{m} \beta_{i}\right\}
$$


Recalling that $\lambda_{n} \longrightarrow 0$ as $n \longrightarrow \infty$, we find that

$$
\frac{1}{\lambda_{n}^{2}}\left\|Q_{n}\right\|_{1, \mu} \leq c \lambda_{n} \int_{\bar{\Omega}}\left(\left|b_{n}\right|+1\right)^{p_{0}} d \mu \longrightarrow 0 \text { as } n \longrightarrow \infty
$$

This result combined with (4.12), (4.15) gives, for $(\alpha, \beta) \in \mathcal{R}$,

$$
\lim _{n \rightarrow \infty} \int_{\bar{\Omega}} k_{\alpha \beta}\left(\sum_{i=1}^{m} b_{n i}\left(\alpha_{i}-\beta_{i}\right)\right)^{2} d \mu=0
$$

Hence

$$
b:=\left(b_{1}, \ldots, b_{m}\right) \in \mathcal{S}^{\perp} .
$$

8. Letting $n \longrightarrow \infty$ in the equation

$$
\tilde{u}_{n i}=\frac{\bar{u}_{i}}{\lambda_{n}}\left(g_{i}\left(\log \left(a_{n i}\right)-q_{i} v_{n 0}-\bar{v}_{i}\right)-g_{i}\left(\log \left(a_{i}\right)-q_{i} v_{0}-\bar{v}_{i}\right)\right)
$$

we find

$$
\tilde{u}_{i}=\bar{u}_{i} g_{i}^{\prime}\left(\log \left(a_{i}\right)-q_{i} v_{0}-\bar{v}_{i}\right)\left(2 b_{i}-q_{i} \tilde{v}_{0}\right)=\bar{u}_{i} g_{i}^{\prime}\left(v_{i}-\bar{v}_{i}\right)\left(2 b_{i}-q_{i} \tilde{v}_{0}\right)
$$

This relation will be used in the next step of the proof.

9. The equations satisfied by $v_{n 0}$ and $v_{0}$, respectively, imply that, for some $\gamma>0$,

$$
\begin{aligned}
& \gamma\left\{\left\|v_{n 0}-v_{0}\right\|_{H^{1}}^{2}+\int_{\bar{\Omega}}\left(e_{0}\left(\cdot, v_{n 0}\right)-e_{0}\left(\cdot, v_{0}\right)\right)\left(v_{n 0}-v_{0}\right) d \mu\right\} \\
& \leq\left\langle E_{0} v_{n 0}-E_{0} v_{0}, v_{n 0}-v_{0}\right\rangle=\sum_{i=1}^{m} \int_{\bar{\Omega}} q_{i}\left(u_{n i}-u_{i}\right)\left(v_{n 0}-v_{0}\right) d \mu .
\end{aligned}
$$

Dividing by $\lambda_{n}^{2}$ and passing to the limit as $n \longrightarrow \infty$, we obtain,

$$
\gamma\left\|\widetilde{v}_{0}\right\|_{H^{1}}^{2} \leq \sum_{i=1}^{m} \int_{\bar{\Omega}} q_{i} \tilde{u}_{i} \tilde{v}_{0} d \mu
$$

Using (4.14), (4.16), and (4.17) we derive from the preceding inequality that

$$
\gamma\left\|\tilde{v}_{0}\right\|_{H^{1}}^{2} \leq \sum_{i=1}^{m} \int_{\bar{\Omega}} \tilde{u}_{i}\left(q_{i} \tilde{v}_{0}-2 b_{i}\right) d \mu=-\sum_{i=1}^{m} \int_{\bar{\Omega}} \bar{u}_{i} g_{i}^{\prime}\left(v_{i}-\bar{v}_{i}\right)\left(q_{i} \tilde{v}_{0}-2 b_{i}\right)^{2} d \mu \leq 0 .
$$

Hence $\tilde{v}_{0}=0, b=0$, and $\tilde{u}=0$.

10. Dividing (4.18) by $\lambda_{n}^{2}$ we find that the sequence $\left(\tilde{v}_{n 0}\right)$ converges strongly in $H^{1}(\Omega)$ to $\tilde{v}_{0}=0$. Moreover, we obtain

$$
\lim _{n \rightarrow \infty} \int_{\bar{\Omega}} \frac{1}{\lambda_{n}}\left(e_{0}\left(\cdot, v_{n 0}\right)-e_{0}\left(\cdot, v_{0}\right)\right) \tilde{v}_{n 0} d \mu=0
$$

By (4.13)

$$
\left|\tilde{u}_{n i}\right| \leq c\left(\sqrt{a_{n i}}+1\right)\left|b_{n i}\right|+c\left(a_{n i}+1\right)\left|\tilde{v}_{n 0}\right| .
$$


Hence $\tilde{u}_{n} \longrightarrow 0$ in $L^{p}\left(\bar{\Omega}, \mu ; \mathbb{R}^{m+1}\right)$ for every finite $p$. By definition of $\lambda_{n}$ (cf. (4.11)) and Lemma 3.7

$$
1=\frac{1}{\lambda_{n}^{2}}\left(F\left(u_{n}\right)-F\left(u^{*}\right)\right) \leq c\left(\left\|\tilde{v}_{n 0}\right\|_{H^{1}}^{2}+\sum_{i=1}^{m}\left\|\tilde{u}_{n i}\right\|_{2, \mu}^{2}\right)+\int_{\bar{\Omega}} \frac{1}{\lambda_{n}}\left(e_{0}\left(\cdot, v_{n 0}\right)-e_{0}\left(\cdot, v_{0}\right)\right) \tilde{v}_{n 0} d \mu .
$$

Because of the preceding results the right hand side converges to 0 as $n \longrightarrow \infty$. This contradiction shows that the assumption made in the beginning of the second step of the proof was wrong, i.e., (4.3) holds, and the proof is complete.

The estimate of the free energy by the dissipation rate of Theorem 4.2 can be used to prove the exponential decay of the free energy to its equilibrium value along any trajectory of the problem (2.13).

Theorem 4.3. Let $(\mathrm{A} 1)-(\mathrm{A} 11)$ be satisfied, let $(u, v)$ be a solution to the initial value problem (2.13), and let $u^{*}$ have the same meaning as in Section 3. For the initial value $u^{0}$ we suppose that $F\left(u^{0}\right)<R_{\mathcal{M}}$. Then there exists $\lambda>0$ such that, for $t \geq \tau \geq 0$,

$$
F(u(t))-F\left(u^{*}\right) \leq \exp (-\lambda(t-\tau))\left(F(u(\tau))-F\left(u^{*}\right)\right) .
$$

Proof. If $(u, v)$ is a solution to $(2.13)$, then $v(t)=E^{-1} u(t) \in \partial F(u(t))$ for a.e. $t \in \mathbb{R}_{+}$, and for $\lambda \in \mathbb{R}$ we obtain (cf. Brézis [1], Lemma 3.3)

$$
\begin{aligned}
& \exp (\lambda t)\left(F(u(t))-F\left(u^{*}\right)\right)-\exp (\lambda \tau)\left(F(u(\tau))-F\left(u^{*}\right)\right) \\
& =\int_{\tau}^{t} \exp (\lambda s)\left\{\lambda\left(F(u(s))-F\left(u^{*}\right)\right)+\left\langle u^{\prime}(s), v(s)\right\rangle\right\} d s \\
& =\int_{\tau}^{t} \exp (\lambda s)\left\{\lambda\left(F(u(s))-F\left(u^{*}\right)\right)-\langle A(v(s)), v(s)\rangle\right\} d s \\
& =\int_{\tau}^{t} \exp (\lambda s)\left\{\lambda\left(F(u(s))-F\left(u^{*}\right)\right)-\rho(v(s))\right\} d s .
\end{aligned}
$$

Setting $\lambda=0, \tau=0$ in (4.19) we find

$$
\forall t \in \mathbb{R}_{+}: \quad F(u(t)) \leq F\left(u^{0}\right)<R_{\mathcal{M}} .
$$

Since $v(s) \in V, u(s)=E v(s) \in U+u^{0}$ for a.e. $s \in \mathbb{R}_{+}$we conclude by Theorem 4.2 that

$$
F(u(s))-F\left(u^{*}\right) \leq C \rho(v(s)) \quad \text { for a.e. } s \in \mathbb{R}_{+} .
$$

Using now (4.19) with $\lambda=1 / C$ we complete the proof.

Finally we want to comment the hypotheses under which we proved Theorem 4.2.

Remark 4.4. In all cases of practical relevance we are aware of in semiconductor technology the set $\mathcal{M}$ defined in (4.1) is empty. In these cases $R_{\mathcal{M}}=+\infty$, and the assumption $F\left(u^{0}\right)<R_{\mathcal{M}}$ means no restriction, i.e., Theorem 4.3 gives a global asymptotic stability result. If $\mathcal{M}$ is not empty we can prove the exponential decay of the free energy only for initial values $u^{0}$ near the equilibrium state $u^{*}$. In that case Theorem 4.3 contains at least a result on local asymptotic stability. 
Remark 4.5. Theorem 4.2 remains true if the reaction coefficients $k_{\alpha \beta}$ depend continuously on the electrostatic potential. This means that $k_{\alpha \beta}$ in the definition of the operator $A$ (cf. (2.8)) is to be replaced by $k_{\alpha \beta}\left(\cdot, v_{0}\right)$ and instead of (A8) we have to assume that

$$
\begin{aligned}
& k_{\alpha \beta}: \bar{\Omega} \times \mathbb{R} \longrightarrow \mathbb{R}_{+} \text {is such that } \\
& k_{\alpha \beta}(x, \cdot) \in C(\mathbb{R}) \text { for } \mu \text {-almost every } x \in \Omega, \\
& k_{\alpha \beta}(\cdot, s) \in L_{+}^{\infty}(\bar{\Omega}, \mu) \backslash\{0\} \text { for every } s \in \mathbb{R} .
\end{aligned}
$$

(It is not necessary to impose a growth condition on $k_{\alpha \beta}$, if the domain of definition of $A$ is modified slightly.) One may also allow $k_{\alpha \beta}$ to depend on the potentials $v_{1}, \ldots, v_{m}$ if only the values of $k_{\alpha \beta}$ can be estimated independently of these potentials.

Remark 4.6. One could admit more general functions $e_{1}, \ldots, e_{m}$ than those described by (2.11) and (A5). We don't go into details here because all functions we met in applications satisfy our hypotheses.

Remark 4.7. We could treat also systems where in addition to the diffusion in $\Omega$ other diffusion processes take place on the surface $\partial \Omega$ or on interfaces because the additional processes lead to an increase of the dissipation rate.

\section{References}

[1] H. Brézis, Opérateurs maximaux monotones et semi-groupes de contractions dans les espaces de Hilbert, Mathematics Studies 5, North-Holland, Amsterdam London, 1973.

[2] A. Glitzky, K. Gröger, and R. Hünlich, Existence, uniqueness and asymptotic behaviour of solutions to equations modelling transport of dopants in semiconductors, in: Special topics in semiconductor analysis (Eds. J. Frehse, H. Gajewski), Bonner Mathematische Schriften 258 (1994), 49-78.

[3] A. Glitzky, K. Gröger, and R. Hünlich, Discrete-time methods for equations modelling transport of foreign-atoms in semiconductors, Nonlinear analysis, to appear.

[4] A. Glitzky and R. Hünlich, Energetic estimates and asymptotics for electro-reactiondiffusion systems, Preprint 168, Weierstraß-Institut für Angewandte Analysis und Stochastik, Berlin, 1995.

[5] K. Gröger, Free energy estimates and asymptotic behaviour of reaction-diffusion processes, Preprint 20, Institut für Angewandte Analysis und Stochastik, Berlin, 1992.

[6] K. Gröger, Boundedness and continuity of solutions to second order elliptic boundary value problems, Nonlinear Analysis 26 (1996), 539-549.

[7] A. Höfler and N. Strecker, On the coupled diffusion of dopants and silicon point defects, Technical Report 94/11, ETH Integrated Systems Laboratory, Zurich, 1994. 
[8] R. V. Kohn, New integral estimates for deformations in terms of their nonlinear strains, Arch. Rat. Mech. Anal. 78 (1982), 131-172.

[9] W. G. Mazja, Sobolev spaces, Leningrad, 1985 (Russian).

[10] H. G. Othmer, The interaction of structure and dynamics in chemical reaction networks, in: Modelling of Chemical Reaction Systems (Eds. K. H. Ebert, P. Deuflhard, W. Jäger), 2-19, Springer-Verlag, 1981.

[11] N. S. Trudinger, On imbeddings into Orlicz spaces and some applications, J. Math. Mech. 17 (1967), 473-483. 